\title{
Rate and Equilibrium Constants for the Epimerization of the Endothelin Receptor Antagonist J-104,132 in Aqueous Solution
}

\author{
Michelle L. Bray, ${ }^{a}$ Daniela Gorbacheva, ${ }^{a}$ Hossain Jahansouz, ${ }^{a}$ Michael J. Kaufman, ${ }^{*}, a$ \\ Kiyofumi Ishikawa, ${ }^{b}$ Nobuo Harada, ${ }^{b}$ and Kimimasa Suzuki ${ }^{b}$ \\ Pharmaceutical Research and Development, Merck Research Laboratories, ${ }^{a}$ WP78A-31, West Point, PA 19486, U.S.A. and \\ Pharmaceutical Research and Development, Laboratories for Technology Development, Banyu Pharmaceutical Co., Ltd., \\ 810, Nishijo, Menuma-Machi, Osato-Gun, Saitama 360-0214, Japan. \\ Received September 13, 1999; accepted October 23, 2000
}

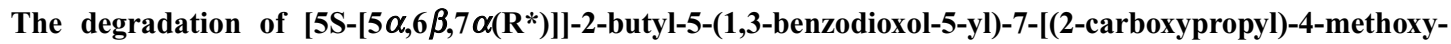
phenyl]-6-dihydro-5H-cyclopenta[b]pyridine-6-carboxylic acid $(\mathrm{J}-104,132)$ was studied in aqueous solution as a function of temperature and $\mathrm{pH}$. The degradation reaction does not proceed to completion; rather, a stable equilibrium is attained in which approximately $2 \%$ of the degradate is produced. Kinetic data for the formation of the degradate are analyzed using an integrated form of the rate law for a reversible first-order reaction, and the forward and reverse rate constants and overall equilibrium constants are presented. Isolation and spectroscopic structural determination indicate that the degradate is the $\mathrm{C} 7 \boldsymbol{\beta}$-epimer of the drug. A mechanism for the epimerization reaction involving a novel enamine-like intermediate is proposed and shown to be consistent with the kinetic data. The rate and equilibrium constants are used to predict the room temperature stability of an injectable formulation of $\mathbf{J}-\mathbf{1 0 4}, \mathbf{1 3 2}$, and these predictions are compared to actual data from long-term stability studies. It is concluded that the preformulation kinetic studies provide essential data needed for optimum drug product development.

Key words stability; kinetics; equilibrium; epimerization; endothelin receptor antagonist; preformulation

An essential part of the pharmaceutical development of new drugs is the quantitative determination of their chemical reactivity under conditions that are relevant to the way they are manufactured, stored, and used. The goals of preformulation stability studies are two-fold. First, it is important to obtain detailed kinetic information under a variety of environmental conditions so that the storage shelf life of a new drug can be estimated. Second, the mechanism of degradation must be determined so that the formulation can be scientifically designed to maximize the stability of the active ingredient in the drug product. Due to the generally higher reactivity of drugs in solution as opposed to the solid phase, preformulation studies are particularly important for compounds that will be formulated as aqueous solutions.

The relatively low reactivity of many substances makes it necessary to conduct high temperature stability studies and extrapolate the results to room temperature. This procedure, however, may be prone to gross inaccuracies under circumstances where the observed reactivity is the sum of several reaction steps such as oxidation reactions. ${ }^{1)}$ Also, extrapolation of high temperature data will lead to errors if there is a change in the reaction mechanism over the temperature range of interest. Thus, a detailed analysis of kinetic, mechanistic, and product data are needed to obtain reliable predictions of room temperature stability for many pharmaceuticals. In this paper, we present the results of preformulation stability studies with [5S-[5 $\left.\left.\alpha, 6 \beta, 7 \alpha\left(\mathrm{R}^{*}\right)\right]\right]$-2-butyl-5-(1,3-benzodioxol-5yl)-7-[(2-carboxypropyl)-4-methoxyphenyl]-6-dihydro-5 $\mathrm{H}$ cyclopenta[b]pyridine-6-carboxylic acid (J-104,132, Fig. 1), a potent and specific non-peptidyl antagonist for the endothelin receptor. ${ }^{2}$ Kinetic and product studies in aqueous solution indicate that $\mathrm{J}-104,132$ is prone to an acid-catalyzed epimerization reaction, and we present a mechanism that involves a novel enamine intermediate. Studies of the reaction as a function of $\mathrm{pH}$ and temperature are presented and analyzed in terms of forward and reverse rate constants for epimerization. Finally, the accelerated stability data are used to derive the room temperature reactivity of the drug in a probe intravenous formulation, and these predictions are compared to long-term data obtained at $25^{\circ} \mathrm{C}$.

\section{Experimental}

Materials All solvents were obtained from Fisher Scientific (Pittsburgh, PA, U.S.A.) and were HPLC grade or equivalent. Reagents were obtained from Fisher Scientific and were certified ACS grade. Laboratory samples of J-104,132 were obtained from Banyu Pharmaceutical Co. Ltd. (Menuma, Japan); the samples were greater than $99 \%$ pure as judged by reverse phase HPLC and were used without further purification.

Kinetic Procedures Solutions used for kinetic studies consisted of the drug $(5.0 \mathrm{mg} / \mathrm{ml})$ and mannitol $(42 \mathrm{mg} / \mathrm{ml}$, used to provide physiologically isotonic media) dissolved in a $0.010 \mathrm{M}$ buffer. The buffers used were sodium citrate/hydrochloric acid $(\mathrm{pH} 6.5$ and 7.5) and tris/hydrochloric acid $(\mathrm{pH}$ 8.5). Measurement of $\mathrm{pH}$ was accomplished with a Fisher Accumet Model $15 \mathrm{pH}$ meter.

The kinetic runs were all performed in essentially the same manner, and a typical run is described below:

A solution of the drug in the mannitol/buffer vehicle was adjusted with $0.01 \mathrm{~m}$ hydrochloric acid to the desired $\mathrm{pH}( \pm 0.1 \mathrm{pH}$ unit), then filtered through a $0.22 \mu \mathrm{m}$ disc filter. The filtrate was filled in $2 \mathrm{ml}$ portions into glass ampoules, and the ampoules were flame sealed. Samples were stored in constant temperature ovens (Thermolyne Series 9000) at 40,60 and $80^{\circ} \mathrm{C}$ $\left( \pm 0.5^{\circ} \mathrm{C}\right)$. At recorded time intervals, samples were removed from storage, allowed to come to room temperature, then quantitatively diluted with deion-

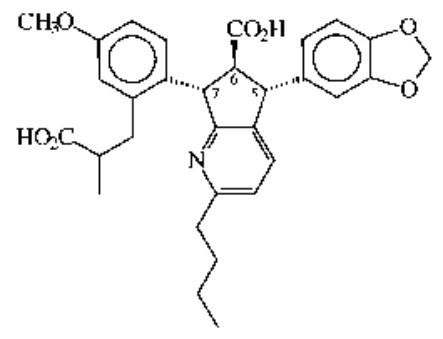

Fig. 1. Chemical Structure of J-104,132 
ized water and assayed for intact drug and degradate levels by HPLC. HPLC analyses were performed on a Waters HPLC system (Model 600 controller and 717 Plus autoinjector equipped with a Model 486 UV detector) using a YMC ODS-AM column $(4.6 \times 250 \mathrm{~mm})$ with UV detection at $230 \mathrm{~nm}$. The compounds were eluted with a linear gradient of 40 to $90 \%$ acetonitrile $v s$. $0.1 \%$ aqueous phosphoric acid over $30 \mathrm{~min}$. The drug elutes with a retention time of $15.4 \mathrm{~min}$, and the degradate elutes at $11.2 \mathrm{~min}$.

In separate experiments, it was shown that the HPLC response factor for $\mathrm{J}-104,132$ and its degradation product are identical to within $\pm 10 \%$. Thus, all calculations of rate and equilibrium constants were performed using HPLC area percent values taken directly from the chromatograms.

Degradate Isolation The chemical structure of the degradation product of J-104,132 was determined by spectroscopic methods following the isolation of an analytically pure sample. The degradate was isolated from stressed $\left(80^{\circ} \mathrm{C} / 2\right.$ weeks) samples of $\mathrm{J}-104,132$ in $0.1 \mathrm{~N}$ hydrochloric acid using preparative scale HPLC. The preparative separation was accomplished on a Waters PrepLC 4000 instrument using an Inertsil Prep-ODS column $(250 \times 50 \mathrm{~mm})$ and a mobile phase consisting of $0.1 \%$ aqueous trifluoroacetic acid and acetonitrile. Using a linear gradient from 30 to $70 \%$ acetonitrile over $90 \mathrm{~min}$ at a flow rate of $25 \mathrm{ml} / \mathrm{min}$, the degradate elutes at $42.0 \mathrm{~min}$ followed by the intact parent compound at $48.4 \mathrm{~min}$. A sample loading of $25 \mathrm{mg}(5 \mathrm{ml} \times 5 \mathrm{mg} / \mathrm{ml})$ per injection was employed. The degradate fractions from 10 injections were combined, most of the acetonitrile and volatile trifluoroacetic acid was removed on a rotary evaporator, and the remaining solution was lyophilized to provide an off-white solid that was shown to be $98.3 \%$ pure by analytical HPLC. The major impurity in the isolated sample was J-104,132.

Proton NMR Spectroscopy ${ }^{1} \mathrm{H}-\mathrm{NMR}$ spectra for J-104,132 and the isolated degradate were acquired on a Varian Unity-500 NMR spectrometer operating at $500.154 \mathrm{MHz}$. The samples for NMR measurement contained approximately $1 \mathrm{mg}$ of solid dissolved in $0.6 \mathrm{ml} \mathrm{CD} \mathrm{CD}_{3} \mathrm{CN}$. All spectra were acquired at $25^{\circ} \mathrm{C}$. Proton chemical shift assignments were made with the combined use of 1D proton, Nuclear Overhauser Effect (NOE) difference ${ }^{3,4)}$ and COSY experiments to determine the structure of the isolated degradate.

Mass Spectrometry The fast atom bombardment mass spectra of J104,132 and the degradate were obtained on a VG-70E magnetic sector mass spectrometer operating at an accelerating potential of $6 \mathrm{kV}$. The analyte $(\mathrm{ca}$. $1 \mathrm{mg}$ ) was dissolved in methanol and diluted with dithiothreotol/dithioerythritol (magic bullet) matrix in methanol. Magnetic scans were performed over the mass/charge range of 800 to 300 at a scan rate of $6 \mathrm{~s} /$ decade.

UV Spectroscopy Ultraviolet spectra were recorded on a Perkin Elmer Lambda 6 spectrophotometer. The samples were dissolved in 50/50 acetonitrile/water at a concentration of $c a .15 \mu \mathrm{g} / \mathrm{ml}$ and spectra were recorded from 190 to $350 \mathrm{~nm}$ at ambient temperature.

\section{Results and Discussion}

Decomposition Kinetics Buffered aqueous solutions of J-104,132 slowly decompose over time to give a single reaction product. The rate of decomposition is a function of the solution temperature and $\mathrm{pH}$, but is not effected by the initial drug concentration. The reaction does not proceed to completion, but rather appears to reach a stable equilibrium state in which approximately $2-3 \%$ of the degradate and $97-$ $98 \%$ of $\mathrm{J}-104,132$ are present. This is shown graphically in Fig. 2, where the degradate level is plotted as a function of time for a kinetic run at $\mathrm{pH}$ 7.5. In particular, the data obtained at $80^{\circ} \mathrm{C}$ show that equilibrium is reached in $c a .96 \mathrm{~h}$, after which no further change in the level of drug or degradate is observed.

Further experimental evidence that an equilibrium exists between the drug and the degradate is provided by kinetic runs performed using the degradate as the starting material. The pure degradate (see next section) was dissolved in the

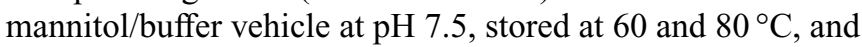
analyzed by HPLC at measured time intervals. At both temperatures, the only product observed following incubation of the degradate under these conditions was J-104,132. Moreover, the final levels of drug and degradate were observed to be the same as found in the kinetic runs starting with $\mathrm{J}$ -

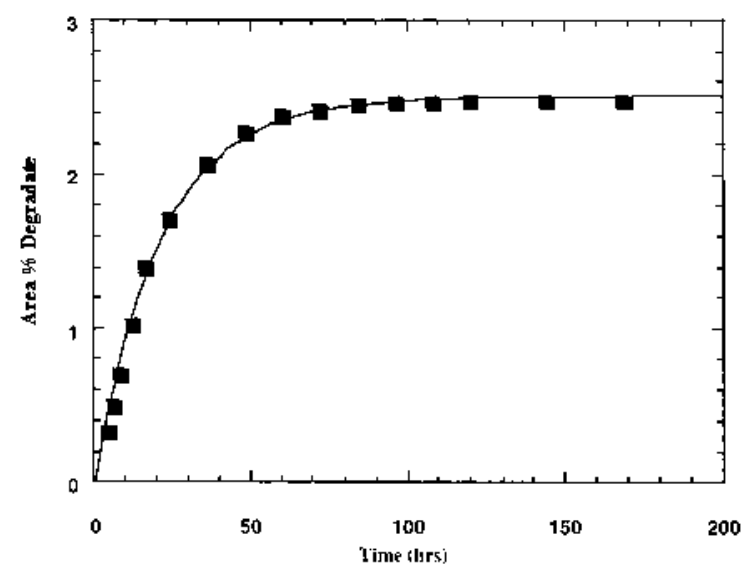

Fig. 2. Plot of Degradate Level (HPLC Area\%) vs. Time for a Kinetic Run at $\mathrm{pH} 7.5$ and $80^{\circ} \mathrm{C}$

Table 1. Rate and Equilibrium Constants for J-104,132 Degradation

\begin{tabular}{ccccccc}
\hline \hline Entry & $T\left({ }^{\circ} \mathrm{C}\right)$ & $\mathrm{pH}$ & $10^{2} k\left(\mathrm{~d}^{-1}\right)$ & $10^{2} K_{\text {eq }}$ & $10^{2} k_{\mathrm{f}}\left(\mathrm{d}^{-1}\right)$ & $10^{2} k_{\mathrm{r}}\left(\mathrm{d}^{-1}\right)$ \\
\hline 1 & 40 & 7.5 & 0.965 & 1.75 & 0.0166 & 0.948 \\
2 & 60 & 7.5 & 12.7 & 2.23 & 0.277 & 12.4 \\
3 & 80 & 7.5 & 111 & 2.56 & 2.77 & 108 \\
$4^{a)}$ & 60 & 7.5 & $18.5^{b)}$ & 2.40 & $0.433^{b)}$ & $18.0^{b)}$ \\
$5^{a)}$ & 80 & 7.5 & $\mathrm{nd}^{c)}$ & 2.47 & $\mathrm{nd}^{c)}$ & $\mathrm{nd}^{c)}$ \\
6 & 60 & 5.5 & 46.8 & 2.53 & 1.15 & 45.6 \\
7 & 60 & 6.5 & 24.0 & 2.34 & 0.549 & 23.5 \\
8 & 60 & 8.5 & 6.76 & 2.15 & 0.142 & 6.62 \\
\hline
\end{tabular}

a) Degradate was the starting material for this run. b) Approximate value based on limited kinetic data. c) nd, not determined.

104,132 as the reactant. This demonstrates that the final concentration of reactant and product is independent of the starting material, and confirms the equilibrium nature of the reaction.

The kinetic data for the formation of the degradate was analyzed using the integrated form of the rate law for a reversible unimolecular reaction: $:^{5}$

$$
\begin{aligned}
& \text { Drug } \underset{k_{\mathrm{r}}}{\stackrel{k_{\mathrm{f}}}{\rightleftharpoons}} \text { Degradate } \\
& \text { [Degradate }]=[\text { Degradate }]_{\mathrm{eq}} \cdot\left(1-e^{-k t}\right)
\end{aligned}
$$

where [Degradate] and [Degradate $]_{\mathrm{eq}}$ represent the concentration of the degradate at time $t$ and at equilibrium, respectively, and $k$ is the observed rate constant for the reaction. The relationship between the overall observed rate constant and the elementary rate constants for the forward reaction $k_{\mathrm{f}}$ and the reverse reaction $k_{\mathrm{r}}$ is given by Eq. 2 . Additionally, the equilibrium constant for the reaction is given by Eq. 3 .

$$
\begin{aligned}
& k=k_{f}+k_{\mathrm{r}} \\
& K_{\mathrm{eq}}=k_{\mathrm{f}} / k_{\mathrm{r}}
\end{aligned}
$$

In practice, the [Degradate] $v s$. time data were fitted to Eq. 1 by non-linear least squares techniques ${ }^{6)}$ using [Degradate $]_{\mathrm{eq}}$ and $k$ as adjustable parameters. The equilibrium constant was obtained from the best fit estimate of [Degradate $]_{\text {eq }}$, and the forward and reverse rate constants were obtained by simultaneous solution of Eqs. 2 and 3. A summary of the kinetic results obtained in this fashion is shown in Table 1. 


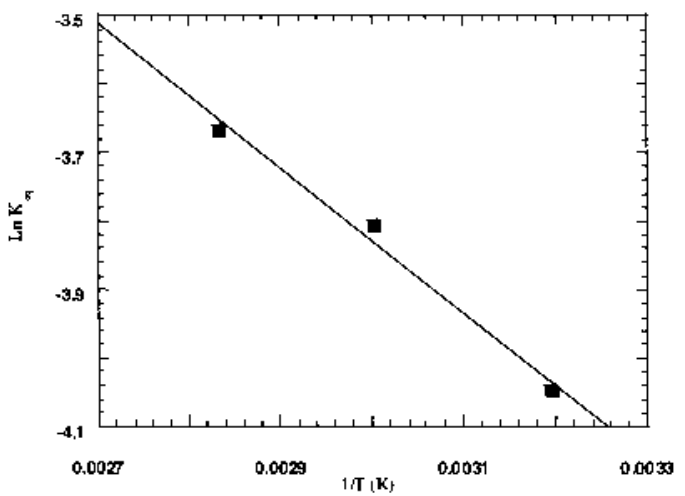

Fig. 3. Temperature Dependence of the Equilibrium Constant for J104,132 Degradation

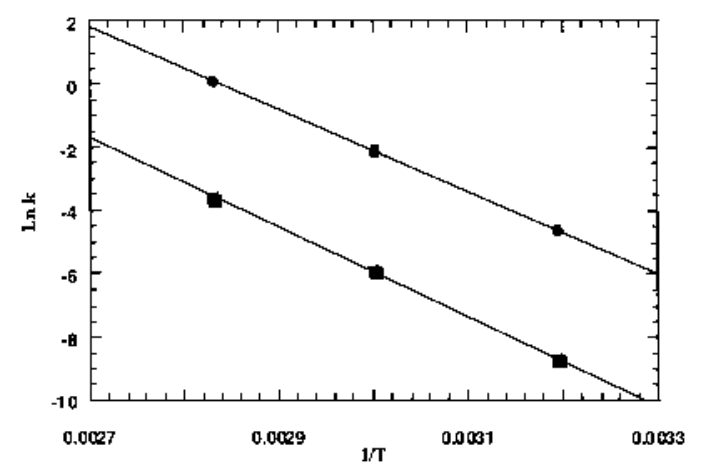

Fig. 4. Temperature Dependence of the Forward (ם) and Reverse Rate Constants for J-104,132 Degradation

The reactions at $\mathrm{pH} 7.5$ were conducted at three temperatures, thus affording the opportunity to obtain additional thermodynamic information. The equilibrium constants were plotted as $\ln K_{\text {eq }} v s$. inverse absolute temperature (Fig. 3), and the derived enthalpy and entropy of reaction are $\Delta H=2.1 \mathrm{kcal} / \mathrm{mol}$ and $\Delta S=1.3 \mathrm{cal} / \mathrm{mol} \cdot \mathrm{K}$. Note that the degradation reaction is enthalpically disfavored but entropically favored. However, the magnitude of the entropy change is quite small and the enthalpy term dominates at temperatures at or near room temperature. It is also notable that the entropy of reaction is quite close to zero, which suggests that the reaction proceeds with no change in the number of products relative to reactants.

The temperature dependence of the forward and reverse rate constants for the reaction were observed to obey the Arrhenius law as shown in Fig. 4. The activation energies for the forward and reverse reactions are $28.1 \mathrm{kcal} / \mathrm{mol}$ and $26.0 \mathrm{kcal} / \mathrm{mol}$, respectively. Thus, although the difference in free energy between the reactant and product molecules is only $2.1 \mathrm{kcal} / \mathrm{mol}$, the kinetic barrier to the reaction is substantially higher.

Degradate Identification The degradation product was isolated by preparative HPLC in quantities sufficient for spectroscopic structure determination. The UV spectrum of the degradate exhibits absorption maxima at 201, 230 and $281 \mathrm{~nm}$, and is identical to that of the parent compound. Mass spectral data indicate no change in the molecular mass for the degradation product relative to the intact drug. Both samples have a molecular mass of 531.3 by mass spectroscopy. The degradate is therefore an isomer of J-104,132.

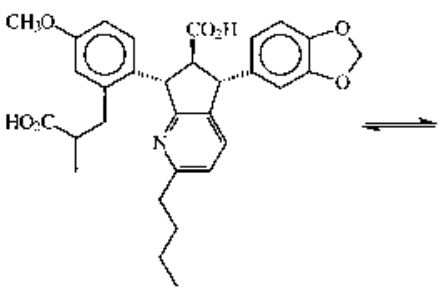

$\mathrm{J}-014,132$

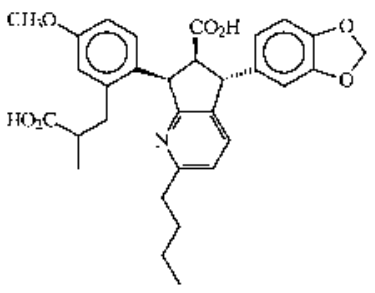

C.7-Epimer
Fig. 5. Degradation of J-104,132 to Form the C7 $\beta$-Epimer Degradate

One-dimensional proton difference NOE experiments of the degradation product with irradiation of H-6 (3.85 ppm) resulted in a strong NOE in $\mathrm{H}-7$ (5.32 ppm) and a weak NOE in $\mathrm{H}-5$ (5.04 ppm), indicating that H-6 and H-7 are cis and H5 and H-6 are trans. Strong NOEs were observed in the 6.75 and $6.82 \mathrm{ppm}$ dioxaindane protons whereas no NOE was observed in the $6.57 \mathrm{ppm}$ methoxyphenyl proton. In addition, a strong NOE was observed in the $6.57 \mathrm{ppm}$ proton upon saturation of $\mathrm{H}-5$ indicating that $\mathrm{H}-5$ and the methoxyphenyl group are in a cis relationship. In the case of $\mathrm{J}-104,132$, only a weak NOE was observed between $\mathrm{H}-5$ and H-6, and H-6 and $\mathrm{H}-7$, indicating that $\mathrm{H}-5$ and $\mathrm{H}-6$ and $\mathrm{H}-6$ and $\mathrm{H}-7$ are trans. In addition, the corresponding NOE observed in the 6.57 ppm proton upon saturation of $\mathrm{H}-5$ was absent for $\mathrm{J}$ 104,132. Thus, the NMR data combined with the UV and MS results indicate that the degradate is the C-7 $\beta$-epimer of the parent drug as shown in Fig. 5.

As discussed in the previous section, the low value of $\Delta S$ for the reaction is consistent with an epimerization reaction that does not change the overall number of reactant and product molecules. The equilibrium is essentially dictated by the higher free energy of the $\beta$-isomer. This is presumably the result of an additional gauche interaction between the C6 and C7 substituents that are $c i s$ to each other in the $\beta$-epimer, but are in the trans configuration in the $\alpha$-epimer. In this regard, it is interesting that the $2.1 \mathrm{kcal} / \mathrm{mol}$ energy difference determined in our studies is quite close to the measured value of $1.7 \mathrm{kcal} / \mathrm{mol}$ between the cis and trans isomers of 1,2-dimethylcyclopentane. ${ }^{7)}$

Aside from the well-known mutarotation of sugars, epimerization is a relatively rare mechanism for the degradation of pharmaceutical agents. Ergotamine has been reported to undergo acid catalyzed epimerization, ${ }^{8)}$ and the activation energy of $24.3 \mathrm{kcal} / \mathrm{mol}$ for that process ${ }^{9)}$ is similar to the value we have determined. Similarly, scopolamine undegoes epimerization at the benzylic site in the molecule; an activation energy of $20.4 \mathrm{kcal} / \mathrm{mol}$ was reported for the base-catalyzed process. ${ }^{10)}$

Mechanism of Epimerization The data presented in entries 2, 6, and 7 of Table 1 indicate that the rate of epimerization is accelerated as the solutions become more acidic, but the extent of epimerization is unaffected by $\mathrm{pH}$. This suggests that the reaction is an acid catalyzed process. Consequently, the mechanism of epimerization is proposed as shown in Fig. 6. The key steps in the sequence are: (1) protonation of the pyridine nitrogen to activate the $\mathrm{C} 7$ hydrogen, (2) deprotonation to form an enamine intermediate with attendant loss of chirality at C7, and (3) reprotonation to generate the product. In this sequence, the formation of the 
<smiles>C[C@H]1[C@@H](C)c2nc([Hg])ccc2[C@@H]1C(=O)O</smiles>

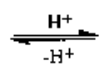<smiles>C[C@@H]1c2nc(P)ccc2[C@@H](C#N)[C@H]1C(=O)O</smiles><smiles>I[C@@H]1C=C[In]1</smiles><smiles>[14CH2]C1c2ccc(Br)nc2C([Al])C1C(=O)O</smiles><smiles>CC1=C2NC(Br)=CC=C2C(C#N)C1C(=O)O</smiles><smiles>C1[In][In]1</smiles><smiles>CC1c2ccc(Br)nc2C(C)C1C(=O)O</smiles>

Fig. 6. Proposed Mechanism for the Acid-Catalyzed Epimerization Reaction

Table 2. Observed and Predicted Levels of the C7-Epimer in an Injectable Formulation

\begin{tabular}{cccc}
\hline \hline$T\left({ }^{\circ} \mathrm{C}\right)$ & Time (weeks) & \% Epimer, observed & \% Epimer, predicted \\
\hline \multirow{2}{*}{25} & 4 & $<0.1$ & 0.05 \\
& 13 & 0.1 & 0.15 \\
& 26 & 0.2 & 0.29 \\
& 39 & 0.3 & 0.41 \\
30 & 52 & 0.4 & 0.52 \\
& 4 & $<0.1$ & 0.11 \\
& 13 & 0.2 & 0.32 \\
& 26 & 0.5 & 0.57 \\
\hline
\end{tabular}

enamine intermediate is undoubtedly the rate-determining step in the reaction, and the acidifying effects of the adjacent pyridinium and aryl groups are required for this step to occur. Note that the effect of solution $\mathrm{pH}$ on the rate is complex since the first step (protonation of the pyridine) is favored by acid but the rate-determining formation of the enamine requires $\mathrm{C} 7$ to act as a carbon acid and is thus basepromoted. Thus, reaction rates are expected to increase with decreasing $\mathrm{pH}$, but less so than the usual ten-fold increase in rate for every 1 unit decrease in $\mathrm{pH}^{11)}$ This is consistent with the kinetic data.
Shelf Life Estimates The kinetic and equilibrium data obtained in the accelerated stability studies can be used to predict the stability of the drug under common storage conditions. For example, the calculated rate and equilibrium constants at $\mathrm{pH} 7.5$ are $k=1.20 \times 10^{-3} \mathrm{~d}^{-1}$ and $K_{\mathrm{eq}}=0.015$ at $25^{\circ} \mathrm{C}$, and $k=5.12 \times 10^{-5} \mathrm{~d}^{-1}$ and $K_{\mathrm{eq}}=0.0116$ at $5^{\circ} \mathrm{C}$. If the amount of degradate that can be tolerated in a pharmaceutical formulation is considered to be $0.5 \%,{ }^{12)}$ then the data indicate that this threshold will be surpassed after 49 weeks of storage at $25^{\circ} \mathrm{C}$. However, due to the large activation energy of the reaction, the temperature dependence is large and the degradate threshold for a formulation stored under refrigerated $\left(5^{\circ} \mathrm{C}\right)$ conditions will not be surpassed for over 30 years.

Subsequent to the preformulation studies described herein, $\mathrm{J}-104,132$ was formulated as a sterile injectable solution containing mannitol and citrate buffer at $\mathrm{pH}$ 7.5. Long term stability data at 5,25 , and $30^{\circ} \mathrm{C}$ were obtained to support the continued use of the product in clinical trials. As predicted, samples stored at $5{ }^{\circ} \mathrm{C}$ (the actual storage condition) showed no detectable epimer levels after 1 year. The actual data obtained in the stability studies at 25 and $30^{\circ} \mathrm{C}$ are shown in Table 2 along with the values predicted from the preformulation studies. It can be seen that the agreement between predicted and actual values is very satisfactory. Thus, the degradation of J-104,132 provides an excellent example of how precise physicochemical analyses can be used to support and optimize pharmaceutical product development.

Acknowledgements The authors would like to acknowledge Drs. Sandor Varga and Harry Ramjit for providing NMR and mass spectral data, respectively. Dr. Joseph Bondi was a great assistance in helping to coordinate the transfer of information between the Merck and Banyu laboratories. We also thank Dr. Orn Almarsson for proof-reading the text and providing helpful suggestions.

\section{References and Notes}

1) Kaufman M. J., Pharm. Res., 7, 289-292 (1990).

2) Nishikibe M., Ohta H., Okada M., Ishikawa K., Hayama T., Fukuroda T., Noguchi K., Saito M., Kanoh T., Ozaki S., Kamei T., Hara K., William D., Kivlighn S., Krause S., Gabel R., Zingaro G., Nolan N., O'Brien J., Clayton F., Lynch J., Pettibone D., Siegl P., J. Pharmacol. Exp. Ther., 289, 1262-1270 (1999).

3) Braunschweiler L., Ernst R. R., J. Magn. Reson., 53, 521-525 (1983).

4) Bax A., Davis D. G., J. Magn. Reson., 65, 355-360 (1995).

5) Moore J. W., Pearson R. G., "Kinetics and Mechanism," 3rd Edition, Wiley-Interscience, New York, 1981. Note that, since the amount of degradate present at $t=0$ in the starting material was essentially zero (below limit of detection), we have used a simplified form of the equations cited in this reference.

6) Curve fitting was performed with KaliedaGraph ${ }^{\mathrm{TM}}$, Version 3.09.

7) Haresnape J. N., Chem. Ind., 1953, 1091-1100.

8) Ott H., Hofmann A., Frey A. J., J. Am. Chem. Soc., 88, 1251-1259 (1966).

9) Kreilgard B., Kisbye J., Arch. Pharm. Chem. Sci. Ed., 2, 38-56 (1974).

10) Smithius L. O. M. J., Pharm. Weekbl., 104, 1097-1104 (1969).

11) van der Houwen O. A. G. J., de Loos M. R., Beijnen J. H., Bult A., Underberg W. J. M., Int. J. Pharm., 155, 137-152 (1997).

12) This value is based on current ICH guidelines as set forth in Q3B: Impurities in New Drug Products, November 1996. 